\title{
Numerical analysis of a dual polarization mode-locked laser with a quarter wave plate
}

\author{
Qiansuo Yang * \\ Key Laboratory of High Temperature Gas Dynamics, Institute of Mechanics, Chinese Academy of Sciences, Beijing 100080, PR China
}

Received 13 October 2003; accepted 28 April 2004

\begin{abstract}
Dynamical behaviors and frequency characteristics of an active mode-locked laser with a quarter wave plate (QWP) are numerically studied by using a set of vectorial laser equation. Like a polarization self-modulated laser, a frequency shift of half the cavity mode spacing exists between the eigen-modes in the two neutral axes of QWP. Within the active medium, the symmetric gain and cavity structure maintain the pulse's circular polarization with left-hand and righthand in turn for each round trip. Once the left-hand or right-hand circularly polarized pulse passes through QWP, its polarization is linear and the polarized direction is in one of the directions of $\pm 45^{\circ}$ with respect to the neutral axes of QWP. The output components in the directions of $\pm 45^{\circ}$ from the mirror close to QWP are all linearly polarized with a period of twice the round-trip time.
\end{abstract}

(c) 2004 Elsevier B.V. All rights reserved.

PACS: 42.60.F; 42.25.J; 42.25.L

Keywords: Polarization; Modulation; Mode-locked; Quarter wave plate; Birefringence; Modal analysis

Without any polarization-selection element in a cavity, the birefringence effect complicates the dynamical behavior of a laser. For example, the output of a VCSEL at a low injection current is linearly polarized in one of its eigen-axes. The increase of the pump current causes the red shift of the wavelength because the refractive index of the gain medium increases with the injection current $[1,2]$ and, therefore, the change of the polarization

\footnotetext{
${ }^{*}$ Tel./fax: +861062553720 .

E-mail address: qsyang@imech.ac.cn (Q. Yang).
}

occurs at certain current level. Another phenomenon is the polarization self-modulation (PSM) which results from the insertion of a quarter wave plate (QWP) into the laser cavity $[3,4]$. This dynamical behavior is also found in some couple cavity lasers such as a VCSEL subject to an optical feedback passing through a QWP [5-11]. Both experimental results and theoretical analysis all reveal that PSM is attributed to the frequency shift between the eigenmodes in the neutral axes of QWP [3,9,12].

If we insert a loss modulator with a modulation frequency of the cavity mode spacing into a laser 
with QWP or a birefringence element (BE), it acts as in an active mode-locked laser. We would like to know whether a mode-locked pulse train (MLPT) is obtained and what about its dynamical behavior? In order to simulate this case accurately and to reveal the corresponding physical mechanism, the longitudinal structure of the cavity has to be included in the numerical analysis. Moreover, the correct equations are required to express the light transmission through QWP or BE and the modulator. The electric field should be expressed as a vector and the gain depends on the polarization of the light in the laser equations. In this paper, the vectorial laser field equations and the mathematical method proposed by Yang et al. [13] are used to analyze the interaction of the light with the population inversion and to explore the dynamical behaviors and the frequency characteristics. In order to assess the validity of the model, our numerical simulation first reproduces the previous experimental results about PSM in a laser

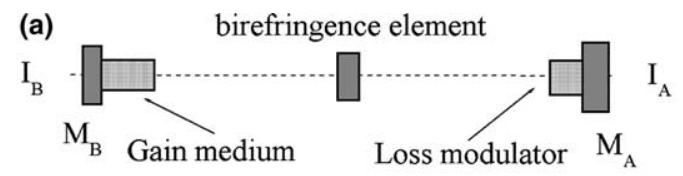

(b) ……... $\mathrm{x}$ - modes, $\quad \mathrm{y}$ - modes

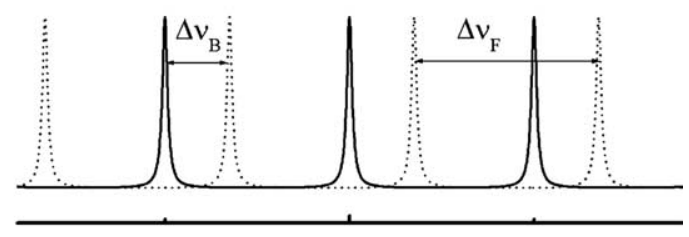

Optical frequency

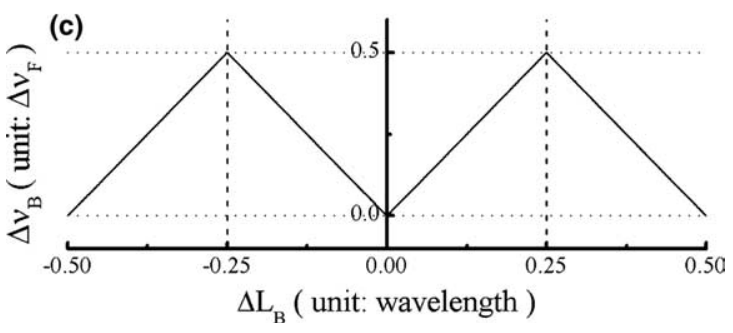

Fig. 1. (a) Schematic of a mode-locked laser with a birefringence element. (b) The mode distribution in the neutral axes. (c) The dependence of the shift, $\Delta v_{\mathrm{B}}$ on the difference of the cavity lengths, $\Delta L_{\mathrm{B}}$. with QWP. The insertion of a loss modulator can force the laser to operate at a mode-locked state. The interesting dynamical behaviors and the corresponding polarization property are analyzed.

Fig. 1(a) shows the schematic structure of a mode-locked laser with a $\mathrm{BE}$ whose neutral axes are set on the eigen-axes of the cavity where the eigen-axes are in $X$ - and $Y$-directions and the longitudinal axis of the cavity is in Z-direction. The loss modulator and the gain medium are close to the two cavity mirrors $M_{\mathrm{A}}$ and $M_{\mathrm{B}}$, respectively. Therefore, the mode-locked pulse can be modulated and amplified continuously when it propagates back and forth in the cavity. The frequency of the modulator is taken as the cavity mode spacing, $\Delta v_{\mathrm{F}}=c / 2 L$ where $L$ is the cavity length.

The following normalized laser equations are used to describe the interaction of the forward and backward vectorial electric fields in the cavity with a homogeneous broadening gain medium [13]

$$
\begin{aligned}
& \frac{\partial E_{x \mathrm{f}}}{\partial t}+v \frac{\partial E_{x \mathrm{f}}}{\partial z}=\int_{0}^{2 \pi} p_{\mathrm{f}} \cos \theta \mathrm{d} \theta-k E_{x \mathrm{f}}, \\
& \frac{\partial E_{y \mathrm{f}}}{\partial t}+v \frac{\partial E_{y \mathrm{f}}}{\partial z}=\int_{0}^{2 \pi} p_{\mathrm{f}} \sin \theta \mathrm{d} \theta-k E_{y \mathrm{f}}, \\
& \frac{\partial E_{x \mathrm{~b}}}{\partial t}-v \frac{\partial E_{x \mathrm{~b}}}{\partial z}=\int_{0}^{2 \pi} p_{\mathrm{b}} \cos \theta \mathrm{d} \theta-k E_{x \mathrm{~b}}, \\
& \frac{\partial E_{y \mathrm{~b}}}{\partial t}-v \frac{\partial E_{y \mathrm{~b}}}{\partial z}=\int_{0}^{2 \pi} p_{\mathrm{b}} \sin \theta \mathrm{d} \theta-k E_{y \mathrm{~b}}, \\
& \frac{\partial p_{\mathrm{f}}}{\partial t}=\left(E_{x \mathrm{f}} \cos \theta+E_{y \mathrm{f}} \sin \theta\right) D-\gamma_{\perp} p_{\mathrm{f}}, \\
& \frac{\partial p_{\mathrm{b}}}{\partial t}=\left(E_{x \mathrm{~b}} \cos \theta+E_{y \mathrm{~b}} \sin \theta\right) D-\gamma_{\perp} p_{\mathrm{b}}, \\
& \frac{\partial D}{\partial t}=R-\gamma_{\|} D-\sum_{\delta=\mathrm{f}, \mathrm{b}}\left[\left(E_{x \delta} \cos \theta+E_{y \delta} \sin \theta\right) p_{\delta}^{*}+\text { c.c. }\right],
\end{aligned}
$$

where $E_{x \mathrm{f}}, E_{y \mathrm{f}}, E_{x \mathrm{~b}}$, and $E_{y \mathrm{~b}}$ are the forward and backward components of the electric fields, respectively. $p_{\mathrm{f}}$ and $p_{\mathrm{b}}$ are the polarization of the gain medium. $D$ and $R$ are the angular distribution of population inversion and the pump rate. $\gamma_{\|}$and $\gamma_{\perp}$ are the longitudinal and transverse relaxation rates of the gain medium. $v$ and $k$ are the light speed and the propagation loss in the gain medium. In the following numerical process, both the 
gain and the pump are considered isotropic in the transverse plane. Meanwhile, the gain and the pump are assumed to be uniform in $Z$-direction for simplification.

After a BE is inserted into a Fabry-Perot cavity, the eigen-modes of the laser splits into two series of longitudinal modes with a frequency shift of $\Delta v_{\mathrm{B}}$ by considering the difference of the cavity lengths in the neutral axes of $\mathrm{BE}, \Delta L_{\mathrm{B}}$, as shown in Fig. 1(b). The dependence of $\Delta v_{\mathrm{B}}$ on $\Delta L_{\mathrm{B}}$ is shown in Fig. 1(c). $\Delta v_{\mathrm{B}}$ reaches its maximum value of $\Delta v_{\mathrm{F}} / 2$ at $\Delta L_{\mathrm{B}}=(2 n+1) \lambda / 4$, where $\lambda$ is the laser wavelength and $n$ is an integer. On the other hand, the number of the modes on the neutral axes depends on the gain linewidth $\Delta v_{\mathrm{G}}$ for both homogeneous and inhomogeneous broadening. First, the multi-mode oscillation is formed if $\Delta v_{\mathrm{G}}>\Delta v_{\mathrm{F}}$. Second, $\Delta v_{\mathrm{G}} \geqslant \Delta v_{\mathrm{B}}$ and $\Delta v_{\mathrm{G}} \leqslant \Delta v_{\mathrm{F}}$ corresponds to the single-mode state in both neutral axes [9]. Finally, only one mode in one of the neutral axes is limited at $\Delta v_{\mathrm{G}}<\Delta v_{\mathrm{B}}$. This is the reason for the linearly polarized output of a VCSEL with a low injection current.

The modulated transmittance of the loss modulator can be written as
$T_{\mathrm{m}}=T_{0}\left[1-A_{\mathrm{m}}\left(1-\sin 2 \pi \Delta v_{\mathrm{F}} t\right)\right]$,

where $T_{0}$ and $A_{\mathrm{m}}$ are the transmittance of the electric field and the modulation depth, respectively. After considering the other loss factors such as the tilted interfaces of two media, which will cause the slight difference of the two losses in the neutral axes, the equivalent reflectance of the electric field for the mirror $M_{\mathrm{A}}$ and these losses are taken as: $r_{\mathrm{A} x}=0.949$ and $r_{\mathrm{A} y}=0.946$. Meanwhile, if the phase shifts caused by the BE in the neutral axes are defined as $\theta_{\mathrm{B}}$ and $-\theta_{\mathrm{B}}$ after one roundtrip, the corresponding reflectance for the combination of the losses $r_{\mathrm{A} x}$ and $r_{\mathrm{A} y}$ and the $\mathrm{BE}$ can be written as

$r_{\mathrm{Ax}}^{\prime}=r_{\mathrm{A} x} \exp \left(-\mathrm{i} \theta_{\mathrm{B}}\right)$
$r_{\mathrm{A} y}^{\prime}=r_{\mathrm{A} y} \exp \left(\mathrm{i} \theta_{\mathrm{B}}\right)$

and $\theta_{\mathrm{B}}=\pi / 2$ for a QWP. The reflectance of mirror $M_{\mathrm{B}}$ and the cavity length are taken as: $r_{\mathrm{B} x}=r_{\mathrm{B} y}=0.99$ and $L=0.6 \mathrm{~m}$. The modulator parameters, $T_{0}$ and $A_{\mathrm{m}}$, are taken as: 1.0 and 0.1 , respectively. If we use a semiconductor amplifier as the gain medium, the typical parameters are as
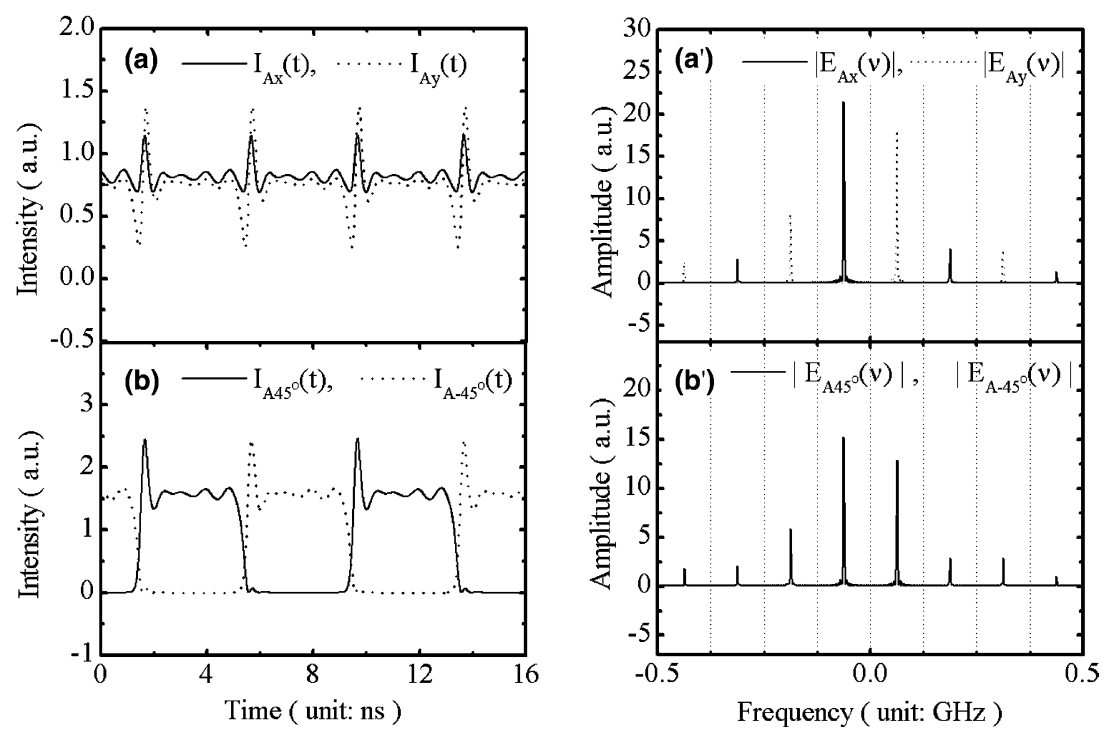

Fig. 2. The intensity waveforms and the spectra of the output components from mirror $M_{\mathrm{A}}$ : (a) and ( $\mathrm{a}^{\prime}$ ) in the neutral axes of the QWP; (b) and $\left(b^{\prime}\right)$ in the directions of $\pm 45^{\circ}$ with respect to the neutral axes in the polarization self-modulation state without the loss modulator. 
follows: $\gamma_{\|}=1 \mathrm{~ns}^{-1}, L_{\mathrm{M}}=1.5 \mathrm{~mm}$, and $k=0.001$, where $L_{\mathrm{M}}$ is the optical length of the gain medium. Since transverse relaxation rate is inversely proportional to the homogeneous broadening, the single-mode state on both neutral axes corresponds to $\Delta v_{\mathrm{G}} \geq \Delta v_{\mathrm{B}}$, and the line-width of the broadband spectrum of a frequency-shift feedback semiconductor laser is about $5 \mathrm{GHz}$ [14]. It is reasonable that $\gamma_{\perp}$ is taken to be $10 \mathrm{~ns}^{-1}$ for a semiconductor medium according to the actual experimental results $[1,8,14]$. The pump threshold of the above system is about 19.5 from our simulation and the pump $R$ is taken as 80 in the following analysis.

In order to assess the validity of the model, we simulated the PSM state in the laser with a QWP
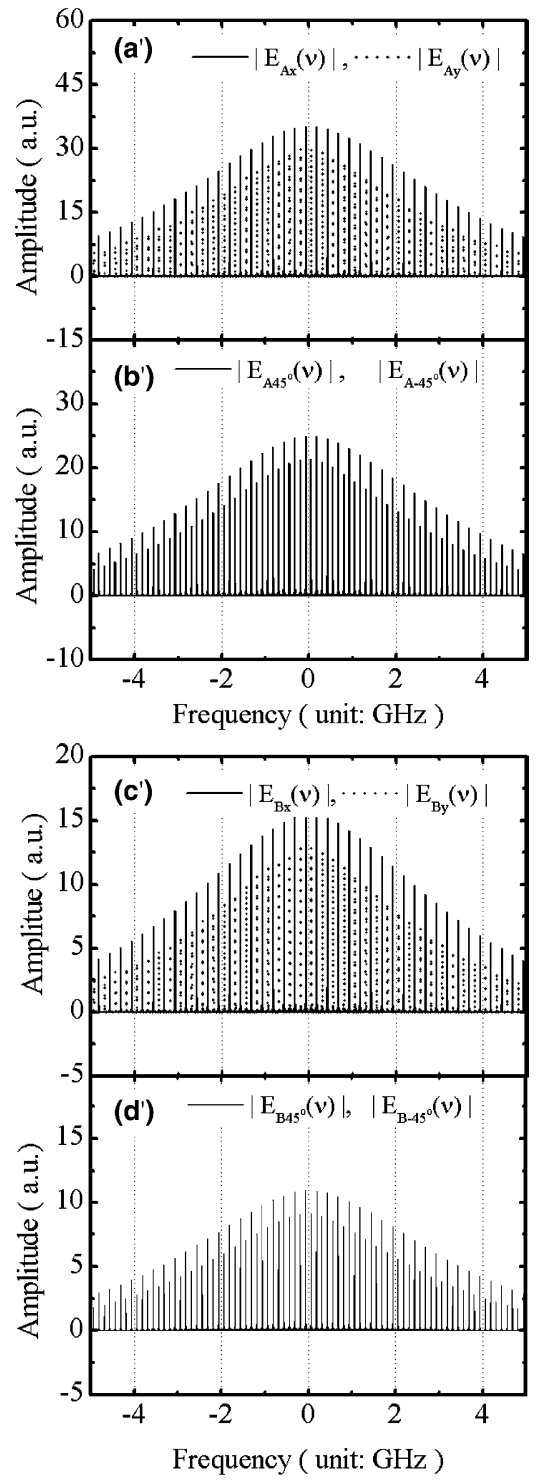

Fig. 3. The intensity waveforms and the spectra of the output components from the two cavity mirrors: (a) and ( $\left.\mathrm{a}^{\prime}\right)$ in the neutral axes of the QWP and from $M_{\mathrm{A}}$; (b) and $\left(\mathrm{b}^{\prime}\right)$ in the directions of $\pm 45^{\circ}$ with respect to the neutral axes of the QWP and from $M_{\mathrm{A}}$; (c) and ( $\mathrm{c}^{\prime}$ ) in the neutral axes and from $M_{\mathrm{B}} ;(\mathrm{d})$ and $\left(\mathrm{d}^{\prime}\right)$ in the directions of $\pm 45^{\circ}$ and from $M_{\mathrm{B}}$. 
but without the loss modulator. Numerical results are shown in Fig. 2. As revealed by the previous experiment [3,9], a frequency shift of $\Delta v_{\mathrm{F}} / 2$ exists among the longitudinal modes in the neutral axes of the QWP. The intensity waveforms from mirror $M_{\mathrm{A}}$ are slightly different due to the difference of $r_{\mathrm{A} x}$ and $r_{\mathrm{A} y}$ in Fig. 2(a $\left.\mathrm{a}^{\prime}\right)$ and (a). In the directions of $\pm 45^{\circ}$, PSM effect is obvious and the two optical spectra are identical, as shown in Fig. 2(b) and $\left(b^{\prime}\right)$. Our simulation also reveals that PSM in Fig. 2(b) gradually becomes the sinusoidal modulation and the multi-mode spectra in Fig. 2(a') degenerate into a single mode in the neutral axis as the cavity length decreases. Once the cavity length $L$ is less than $6 \mathrm{~cm}$, the single longitudinal mode spectrum and the stable outputs in the neutral axes can be obtained because $\Delta v_{F}$ is close to the homogeneous broadening being about $\gamma_{\perp} / \pi$, which corresponds to the experimental results of VCSEL $[1,8,9]$.

Fig. 3 shows the intensity waveforms and the optical spectra of the MLPTs from mirrors $M_{\mathrm{A}}$ and $M_{\mathrm{B}}$ after the loss modulator is added. In the neutral axes of QWP, the laser acts as two solitary
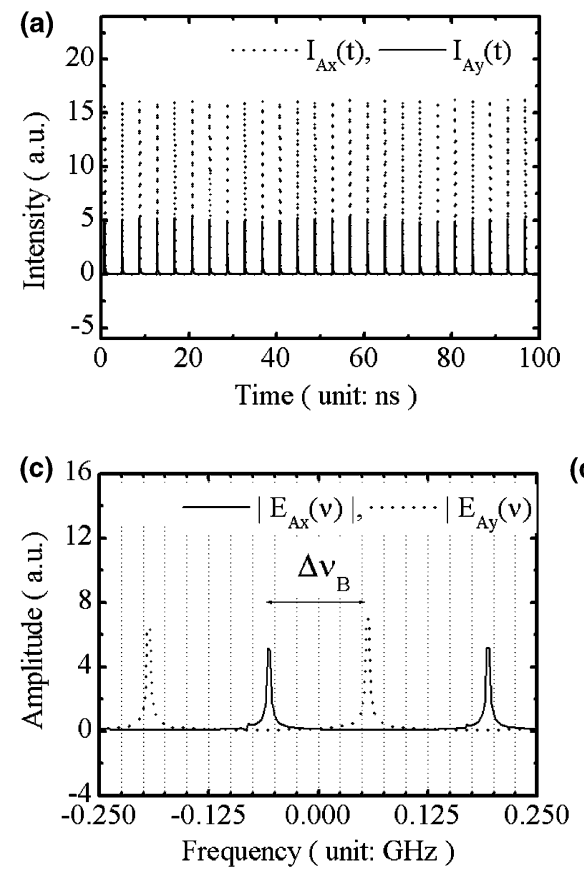

mode-locked lasers and the two MLPTs are in phase due to the common modulator, as shown in Fig. 3(a) and (c), and the difference of the peaks from mirror $M_{\mathrm{A}}$ attributes to the difference of the losses $r_{\mathrm{A} x}$ and $r_{\mathrm{A} y}$. A shift of $\Delta v_{\mathrm{F}} / 2$ between the longitudinal modes is shown in Fig. 3( $\left.\mathrm{a}^{\prime}\right)$ and $\left(\mathrm{c}^{\prime}\right)$. Since the optical spectra in the directions of $\pm 45^{\circ}$ are the vector mapping of the two spectra in the neutral axes, there is a frequency separation of $\Delta v_{\mathrm{F}} / 2$ for both of them due to the frequency shift, as shown in Fig. $3\left(b^{\prime}\right)$ and $\left(d^{\prime}\right)$. Like a polarization self-modulated laser, in the directions of $\pm 45^{\circ}$, the MLPTs from mirror $M_{\mathrm{A}}$ are all with a period of twice the round-trip time and at the antiphase state in Fig. 3(b). However, the output components from mirror $M_{\mathrm{B}}$ are all with a period of one roundtrip time and in phase in Fig. 3(d).

The above dynamical behaviors can be explained by the synchronous back and forth propagation of mode-locked pulses in two neutral axes of the QWP in the cavity, as shown in Fig. 3(a) and (c). The vector superposition of two mode-locked pulses in the neutral axes of the QWP is linearly polarized in the directions of $45^{\circ}$ or $-45^{\circ}$ when it is
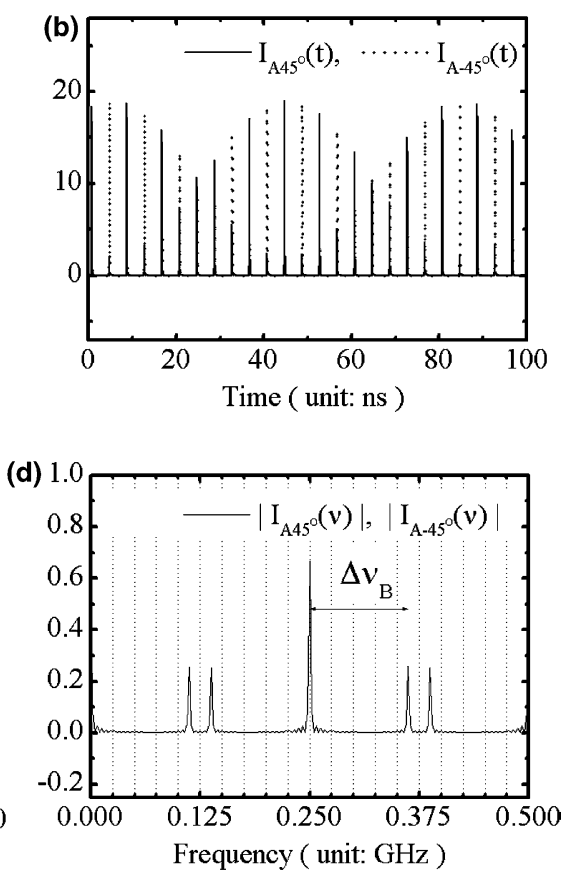

Fig. 4. (a) and (b) The intensity waveforms of the output from mirror $M_{\mathrm{A}}$ in the neutral axes of the QWP and the directions of $\pm 45^{\circ}$ with respect to the neutral axes of the QWP. (c) and (d) The optical spectra and the power spectrum for $\Delta L_{\mathrm{B}}=0.9 \lambda / 4$. 
located at the region between the QWP and mirror $M_{\mathrm{A}}$. Having passed through the QWP, it changes into a circular polarized light either left-hand or right-hand. The following amplification of the light by the gain medium does not affect its polarization property due to the circular symmetry of the gain and the pump. After the amplification is finished and it enters the region between QWP and mirror $M_{\mathrm{A}}$, the polarization comes back linearly but in the directions of $-45^{\circ}$ or $45^{\circ}$. Thus, the linear polarization in the directions of $45^{\circ}$ or $-45^{\circ}$ in the region between QWP and mirror $M_{\mathrm{A}}$ and the circular polarization with right-hand or left-hand between QWP and the mirror $M_{\mathrm{B}}$ are all alternate with a period of twice the round-trip time, as shown in Fig. 3(b). The output from $M_{\mathrm{A}}$ is with a period of twice the round-trip time but the output intensity from $M_{\mathrm{B}}$ is with a period of the round-trip time in Fig. 3(d). This explanation is also the same as the laser with an inhomogeneous broadening gain medium since the phase locking among the longitudinal modes is induced by the modulator and does not depend on the population inversion. Further simulation reveals that the approximate symmetry and the adequate length of the cavity are essential for the alternation of the pulse in the directions of $\pm 45^{\circ}$.

When the difference of the cavity lengths in the two neutral axes produced by the BE slightly deviates from the quarter-wave, the MLPTs on the neutral axes are almost the same as with a QWP, as shown in Fig. 4(a) where $\Delta L_{\mathrm{B}}=0.9 \lambda / 4 . \Delta v_{\mathrm{B}}$ in Fig. 4(c) follows the relationship shown in Fig. 1(c). However, a small modulation for the two MLPTs in the neutral axes is in phase and with a frequency of $\left|2 \Delta v_{\mathrm{B}}-\Delta v_{\mathrm{F}}\right|$ due to the amplification of the MLPTs by the common population inversion. In the power spectrum in the directions of $\pm 45^{\circ}$, the sum and difference frequency effects among $\Delta v_{\mathrm{B}}$ and $m \Delta v_{\mathrm{F}}$ can be found in Fig. 4(d) where $m$ is an integer. The complete modulation on the MLPTs is obvious in Fig. 4(b) with the frequency of $\left|2 \Delta v_{\mathrm{B}}-\Delta v_{\mathrm{F}}\right|$.

In summary, our simulation predicts that a mode-locked laser with a QWP and a loss modu- lator can operate at dual polarization states. In the neutral axes of the QWP, the two MLPT are both in phase and there is a frequency shift of half the cavity mode spacing between them. In the directions of $\pm 45^{\circ}$, the two MLPT with a period of twice the round-trip time and at antiphase state can be obtained from one of the cavity mirrors. The dynamical behavior results from the shifting of the longitudinal modes in the neutral axes.

\section{Acknowledgements}

The author acknowledges helpful discussions with Dr. Yanming Liu.

\section{References}

[1] Shijun Jiang, Zeqi Pan, M. Dagenais, Appl. Phys. Lett. 63 (1993) 3545 .

[2] K.D. Choquette, D.A. Richie, R.E. Leibenguth, Appl. Phys. Lett. 64 (1994) 2062.

[3] M. Brunel, M. Vallet, G. Ropars, A. Le Floch, F. Bretenaker, G. joulie', J.-C. Keromnes, Phys. Rev. A 55 (1997) 1391.

[4] M. Vallet, M. Brunel, F. Bretenaker, M. Alouini, A. Le Floch, Appl. Phys. Lett. 74 (1999) 3266.

[5] N. Badr, I.H. White, M.R.T. Tan, Y.M. Houng, S.Y. Wang, Electron. Lett. 30 (1994) 1227.

[6] W.H. Loh, A.T. Schremer, C.L. Tang, IEEE Photonic. Technol. Lett. 2 (1990) 467.

[7] W.H. Loh, Y. Ozeki, C.L. Tang, Appl. Phys. Lett. 56 (1990) 2613

[8] F. Ginovart, F. Robert, P. Besnard, J. Opt. B: Quantum Semiclass. Opt. 1 (1999) 646.

[9] F. Robert, P. Besnard, M.L. Chares, G.M. Stephan, IEEE J. Quantum Electron. 33 (1997) 2231.

[10] G. Ropars, P. Langot, M. Brunel, M. Vallet, F. Bretenaker, A. Le Floch, K.D. Choquette, Appl. Phys. Lett. 70 (1997) 2661.

[11] H. Li, A. Hohl, A. Gavrielides, H. Hou, K.D. Choquette, Appl. Phys. Lett. 72 (1998) 2355.

[12] Q.S. Yang, A. Sasoh, Opt. Commun. 219 (2003) 309.

[13] Q.S. Yang, P.Y. Wang, J.H. Dai, H.J. Zhang, IEEE J. Quantum Electron. 34 (1998) 1135.

[14] A. Yoshizawa, H. Tsuchida, Opt. Commun. 155 (1998) 51. 